\title{
Perfilador de Vento no Centro de Lançamento de Alcântara: Uma análise estatística
}

\author{
The Alcântara Launching Center Wind Profiler: A statistical analysis \\ Diogo M. Custodio, Jorge Yamasaki, Hildo R. Quinsan Jr., Gilberto F. Fisch
}

Novembro de 2015

\begin{abstract}
Resumo
O monitoramento das condições atmosféricas é uma das principais atividades da Meteorologia Aeroespacial. Durante operações de lançamento de veículos espaciais, medir o vento em diversas camadas atmosféricas é fundamental para o sucesso da missão. No Centro de Lançamento de Alcântara opera um Perfilador de Vento (WP) Vaisala LAP-12000 que fornece perfis verticais do vento entre 1500 e $6000 \mathrm{~m}$ de altitude, com medidas em camadas de $145 \mathrm{~m}$, realizadas em intervalos de 10 minutos. Este trabalho apresenta uma análise estatística de um ano de dados obtidos com o WP através de comparação com perfis verticais do vento obtidos por radiossondas (RS) Vaisala RS92SGP. Foram feitas análises de correlação $(\rho)$ da velocidade do vento e suas componentes zonal e meridional; média e o desvio padrão $(\sigma)$ das diferenças entre as componentes zonal e meridional. Observou-se que existe uma forte correlação entre os dados, com $\rho \geqslant 0,75$ e $\sigma \leqslant 3 \mathrm{~m} / \mathrm{s}$ em todas as análises.
\end{abstract}

Palavras-chave: Radiossondagem, perfil vertical do vento, correlação linear.

\begin{abstract}
Monitoring the atmospheric conditions is one of the main activities in Aerospace Meteorology. During the launch spacecraft operations, measuring the wind at various atmospheric layers is crucial to mission success. In the Alcantara Launch Center operates a Wind Profiler (WP) Vaisala LAP-12000 which provides vertical profiles of wind between 1500 and $6000 \mathrm{~m}$ of altitude, in layers of $145 \mathrm{~m}$, at intervals of 10 minutes. This study conducts a statistical analysis over one year of data obtained with WP by comparison with Vaisala RS92SGP radiosondes (RS). Correlation ( $\rho$ ) analyses were performed on wind speed and its zonal and meridional components; and mean and standard deviation $(\sigma)$ of the differences between the zonal and meridional components. The results has shown a strong correlation between the data acquired with both instruments, with $\rho \geqslant 0,75$ and $\sigma \leqslant 3 \mathrm{~m} / \mathrm{s}$ in all performed analyses.
\end{abstract}

Keywords: Radiosonde, vertical wind profile, linear correlation. 


\section{Introdução}

O monitoramento das condições atmosféricas é uma das principais atividades da Meteorologia Aeroespacial. Durante as missões de lançamento de veículos espaciais, medir o vento é fundamental no suporte ao planejamento das operações, ajudando, por exemplo, a definir janelas de lançamento e determinar sua trajetória, garantindo o sucesso da missão (Marques e Fisch, 2005).

No Centro de Lançamento de Alcântara (CLA; $2^{\circ} 18^{\prime} S$, $44^{\circ} 21^{\prime} \mathrm{W}, 21 \mathrm{~m}$ de altitude) as medidas de vento são realizadas através de diferentes técnicas e instrumentos (Fisch et al., 2015), dentre os quais está o Perfilador de Vento (Wind Profiler, WP), modelo LAP-12000, da fabricante Vaisala. Consiste em um conjunto de 144 antenas tipo Yagi-Uda de 3 elementos, que operam em frequências de VHF, variando entre 46 e $68 \mathrm{MHz}$ (Figura 1). Seu funcionamento é baseado nos princípios de um radar Doppler pulsado com feixe orientado verticalmente que fornece informações da velocidade e direção do vento em sucessivas camadas da troposfera (Figura 2).

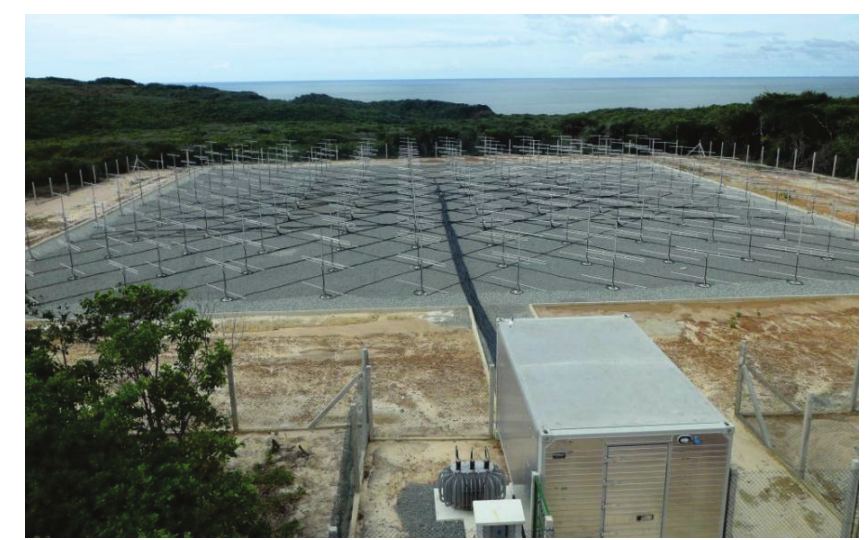

Figura 1: O Perfilador de Vento LAP-120000 instalado no CLA. Fonte: CLA/Divulgação.

Instalado em 2011 e em operação desde o segundo semestre de 2012, trata-se do primeiro WP com uso contínuo na América do Sul. Sua configuração atual fornece perfis verticais do vento entre 1500 e $6000 \mathrm{~m}$ de altitude, com medidas em camadas de $145 \mathrm{~m}$, realizadas em intervalos de 10 minutos, permitindo um acompanhamento contínuo da evolução temporal do perfil vertical do vento, desde a camada de transição até a média troposfera. (Figura 3)

Perfiladores de vento já possuem alguns aspectos gerais documentados (Dibbern et al., 2003); no entanto trabalhos anteriores salientam que não necessariamente estes se aplicam para todas as localidades e estações do ano (Strauch et al., 1987; Weber e Wuertz, 1990; Weber et al., 1990).

A fim de avaliar seu desempenho, este trabalho realiza uma análise estatística de um ano de dados obtidos com o WP através de comparação com perfis verticais do vento obtidos simultaneamente por radiossondagem (RS), utilizando as sondas RS92-SPG e estação receptora Digicora III, ambas da empresa Vaisala. A avaliação através de diagramas de dispersão; coeficientes de correlação de Pearson; médias e desvios padrão; séries temporais e perfis verticais visa expandir e complementar a análise preliminar realizada por Quinsan Jr. et al. (2014).

\section{Desenvolvimento e metodologia}

Utilizou-se um conjunto de um ano de dados, compreendendo o intervalo entre o mês de junho de 2014 e maio de 2015. Neste período foram lançadas 348 radiossondas nos horários das 00h e 12h UTC no CLA. Cada perfil vertical obtido via RS foi processado para se equiparar ao

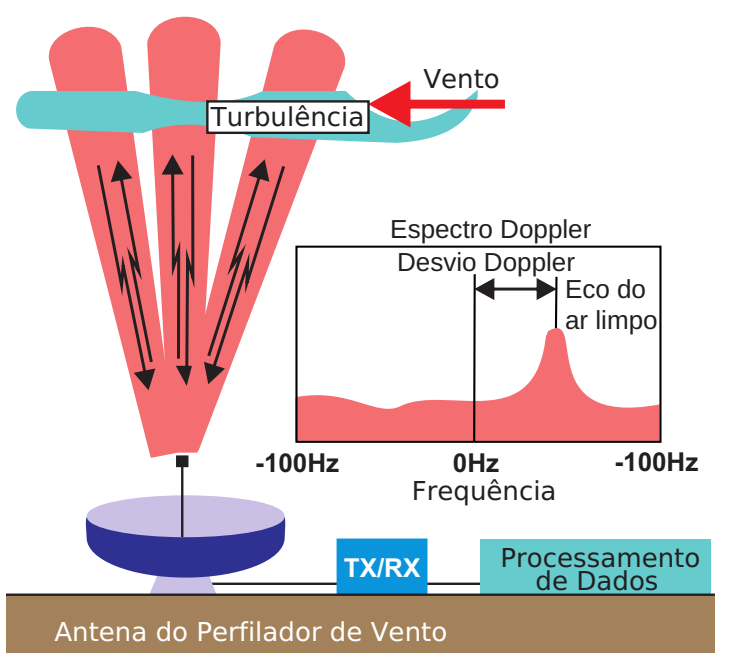

Figura 2: Princípio de funcionamento esquematizado do WP. Feixes pulsados verticalmente são emitidos e detectados. A partir do espectro das ondas refletidas, a direção e velocidade do vento em sucessivas camadas atmosféricas são inferidas. Adaptado de: CLA/Divulgação.

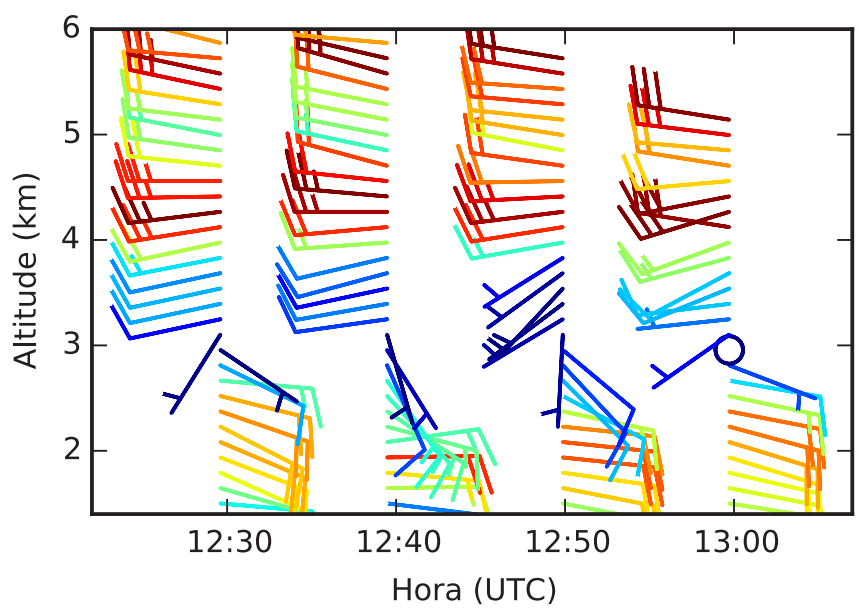

Figura 3: Evolução temporal do perfil do vento no CLA no dia 21/02/2015, entre as 12:30 e 13:00 UTC, em intervalos de 10 minutos, obtida com o WP. 
às $00 \mathrm{~h}$ UTC. Neste período totalizam 166 perfis WPxRS com 5013 pares de pontos de dados (Figura 6). Da análise da velocidade do vento (Figura 6.a) e suas componentes zonal (Figura 6.b) e meridional (Figura 6.c), obtém-se os coeficientes de correlação (a) 0,76, (b) 0,83, (c) 0,80, respectivamente. As diferenças entre as componentes zonal $\left(u_{R S}-u_{W P}\right)$ e meridional $\left(v_{R S}-v_{W P}\right)$ estão representadas na figura 6.d e seus respectivos valores médios e desvios padrão são $-0,3 \pm 2,7 \mathrm{~m} / \mathrm{s}$ e $0,2 \pm 2,4 \mathrm{~m} / \mathrm{s}$.
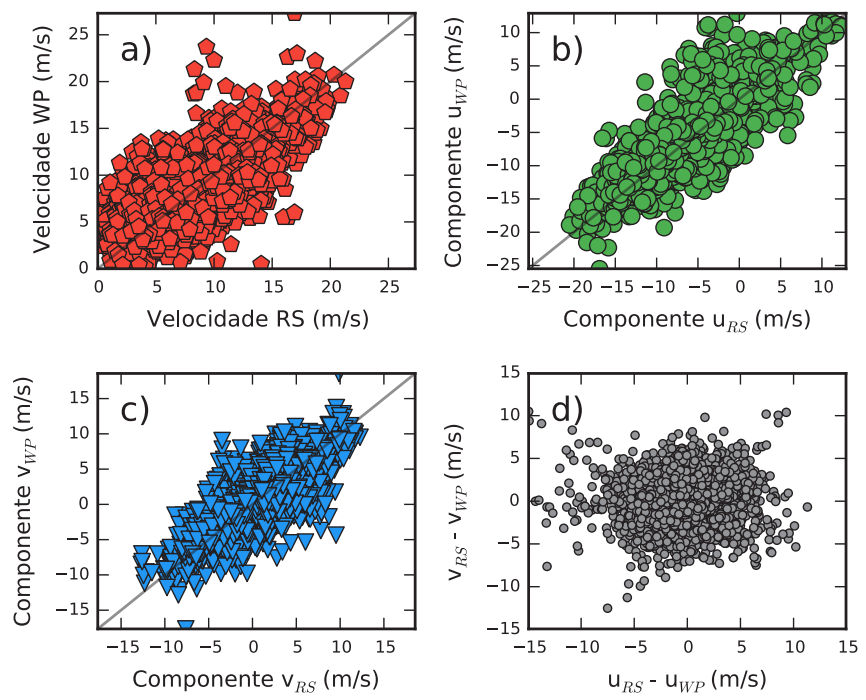

Figura 6: Similar a figura 5, mas para as medidas das 00h UTC.

\subsubsection{Análise no período diurno (12h UTC)}

Similarmente à análise anterior, está selecionado apenas o conjunto de dados correspondente às medições diurnas, realizadas às $12 \mathrm{~h}$ UTC. O total de perfis WPxRS analisados é 172 com 5307 pares de pontos de dados (Figura 7).

Os coeficientes de correlação da velocidade do vento (Figura 7.a) e suas componentes zonal (Figura 7.b) e meridional (Figura 7.c) são, respectivamente, (a) 0,75, (b) 0,84 , (c) 0,80. Na figura 7.d estão representadas as diferenças entre as componentes zonal $\left(u_{R S}-u_{W P}\right)$ e meridional $\left(v_{R S}-v_{W P}\right)$. Os valores médios e desvios padrão são $-0,7 \pm 2,7 \mathrm{~m} / \mathrm{s}$ e $0,2 \pm 2,4 \mathrm{~m} / \mathrm{s}$.

A partir dos resultados obtidos em 2.1.1 e 2.1.2, observa-se que os coeficientes de correlação não possuem uma dependência no ciclo diário, apresentando forte correlação tanto nas medidas das 00h UTC quanto nas medidas das $12 \mathrm{~h}$ UTC. Por isso, as próximas análises serão apresentadas somente para as observações referentes às $12 \mathrm{~h}$ UTC.

\subsection{Análise da correlação com a altura}

Para as atividades aeroespaciais realizadas pelo CLA, é de especial interesse que a qualidade das observações
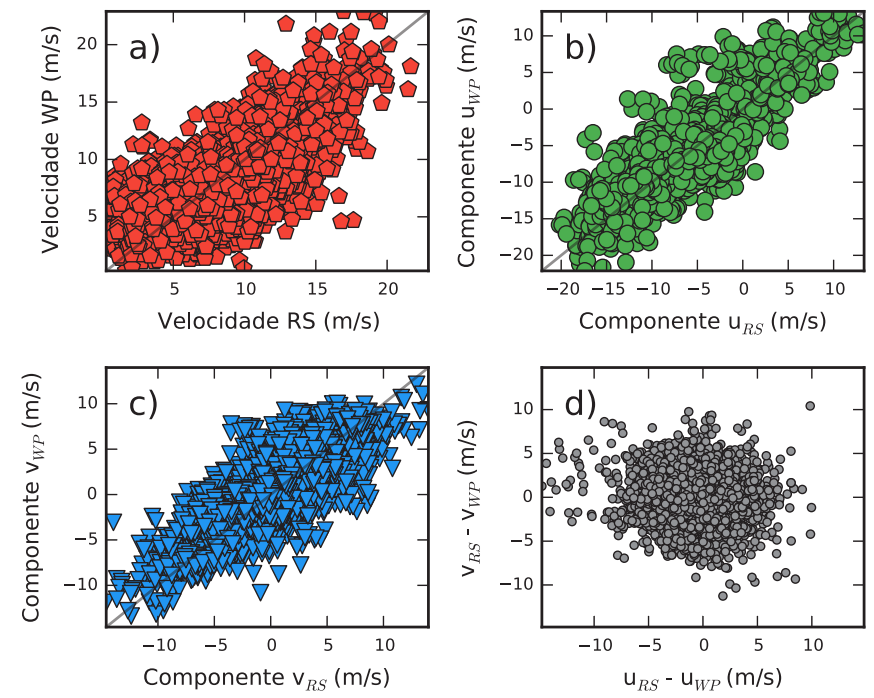

Figura 7: Similar a figura 5, mas para as medidas das 12h UTC.

seja mantida com a altura. Isto será verificado através da análise dos coeficientes de correlação com a altura.

A partir do perfil vertical dos coeficientes de correlação (Figura 8) é possível observar a forte correlação entre as medidas de WP e RS desde o limite inferior até o limite superior do domínio, tanto para a velocidade do vento quanto para suas componentes zonal e meridional.

Os valores dos coeficientes de correlação variam entre 0,70 e 0,80 . O menor valor do coeficiente de correlação é de 0,59 na altura de $2062 \mathrm{~m}$ e, mesmo assim, representa uma correlação de moderada a forte. Observa-se que a correlação apresenta um pequeno aumento com a altura, possivelmente associada com ventos mais intensos e menos variáveis em níveis mais altos.



Figura 8: Perfil vertical da correlação (linhas) e pares de dados WPxRS (barras) entre os níveis de 1500 e 6000m, de $06 / 2014$ a $05 / 2015$ as 12 h UTC, da velocidade do vento e suas componentes zonal e meridional. 


\subsection{Análise sazonal da correlação}

Para verificar se a qualidade das medições possui variações sazonais, foi realizada a avaliação dos coeficientes de correlação mensalmente. Na série temporal, representada na figura 9, observa-se que os valores dos coeficientes de correlação variam entre 0,60 e 0,80 , tanto para a velocidade quanto para suas componentes. Durante SON a correlação variou entre 0,55 e 0,85 e em FMA variou entre 0,46 e 0,95 , tendo o menor valor $(0,46)$ ocorrido em março de 2015. O baixo número de pares de dados analisados nos meses de outubro de 2014 e março de 2015 foram devidos à restrições operacionais e manutenções ocorridas sobre o WP neste período, no CLA, sendo, também, a causa provável da diminuição nos valores de correlação. Não se observa dependência na sazonalidade, mantendo as mesmas características tanto no período seco (SON) quanto no chuvoso (FMA).

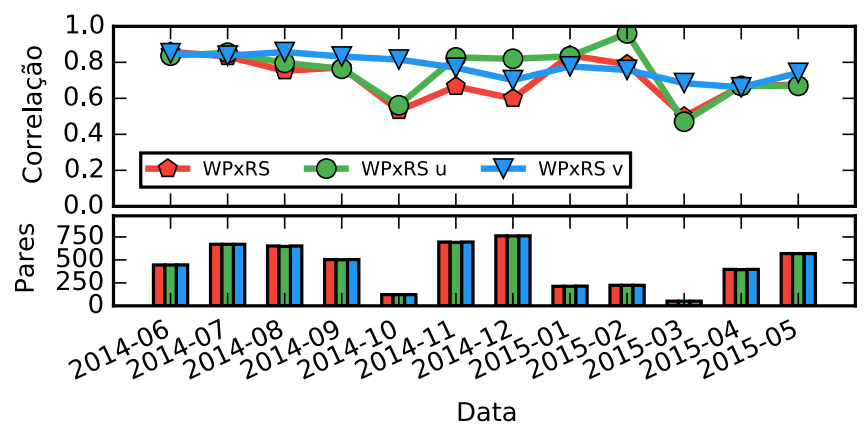

Figura 9: Série temporal dos coeficientes de correlação e pares de dados entre 06/2014 e 05/2015 as 12h UTC. As linhas representam a evolução temporal de $\rho$ e as barras o número de pares WPxRS analisados, para a velocidade do vento e suas componentes zonal e meridional.

\section{Conclusões}

Neste trabalho foi realizada uma análise estatística preliminar entre as medições do WP e RS no CLA. Observouse uma forte correlação entre as medidas de WP e RS, com $\rho$ maior que $0,75 \mathrm{em}$ todas as análises (Tabela 1 ). Verificou-se um pequeno aumento na correlação com a altura e não foi detectada variação significativa com o ciclo diurno ou sazonal, sendo mantida a correlação de moderada a forte entre WP e RS, tanto no período seco quanto no chuvoso. Estes resultados mostram que, em comparação com as RS, o WP apresenta um bom desempenho. O desvio padrão menor que $3 \mathrm{~m} / \mathrm{s}$ mantém a incerteza das medições em nível tolerável para a aplicação ao qual está destinado.

Trabalhos futuros serão realizados investigando quais sistemas atmosféricos que geram valores espúrios em relação a RS. Também será explorada a capacidade de monitorar, de forma praticamente contínua, o perfil ver- tical do vento, tanto com aplicações em pesquisa quanto operacional, durante missões de lançamento.

Tabela 1: Sumário das estatísticas de comparação entre WP e RS entre 06/2014 e 05/2015. São apresentados os coeficientes de correlação $(\rho)$, média $(\mathrm{m} / \mathrm{s})$ e desvio padrão $(\sigma, \mathrm{m} / \mathrm{s})$ da velocidade do vento e das diferenças (WP-RS) entre componentes zonal e meridional.

\begin{tabular}{|c|c|c|c|c|c|c|c|c|c|}
\hline & \multicolumn{3}{|c|}{ Todos } & \multicolumn{3}{|c|}{ 00h UTC } & \multicolumn{3}{|c|}{ 12h UTC } \\
\hline & $\rho$ & Méd. & $\sigma$ & $\rho$ & Méd. & $\sigma$ & $\rho$ & Méd. & $\sigma$ \\
\hline$|\vec{V}|$ & 0,75 & 0,3 & 2,5 & 0,76 & 0,1 & 2,5 & 0,75 & 0,6 & 2,5 \\
\hline$u$ & 0,84 & $-0,5$ & 2,7 & 0,83 & $-0,3$ & 2,7 & 0,84 & $-0,7$ & 2,7 \\
\hline$v$ & 0,80 & 0,2 & 2,4 & 0,80 & 0,2 & 2.4 & 0,80 & 0,2 & 2,4 \\
\hline
\end{tabular}

\section{Referências}

Dibbern, J., Engelbart, D., Goersdorf, U., et al. (2003). Operational Aspects of Wind Profiler Radars. World Meteorological Organization, Instruments and Observing Methods, 79(WMO/TD 1196).

Fisch, G., de Paiva e Melo, E., Yamasaki, J., Custodio, D. M., Couto, V. M., Iriart, P. G., da Silva, A. F. G. (2015). The wind profile at the Alcântara Space Center: observations and numerical modeling applied to the rocket launching. Em: Proceedings of the 14th International Conference on Wind Engineering, Porto Alegre, RS.

Marques, R., Fisch, G. (2005). As atividades de meteorologia aeroespacial no Centro Técnico Aeroespacial (CTA). Boletim da Sociedade Brasileira de Meteorologia, 29(3), 21-25.

Quinsan Jr., H. R., Custodio, D. M., Yamasaki, J., Fisch, G. (2014). Análise preliminar de dados de vento obtidos por um Perfilador de Vento no Centro de Lançamento de Alcântara. Em: Anais do XVIII Congresso brasileiro de meteorologia, Recife, PE.

Strauch, R., Weber, B., Frisch, A., Little, C., Merritt, D., Moran, K., Welsh, D. (1987). The precision and relative accuracy of profiler wind measurements. Journal of Atmospheric and Oceanic Technology, 4(4), 563-571.

Weber, B. L., Wuertz, D. B. (1990). Comparison of rawinsonde and wind profiler radar measurements. Journal of Atmospheric and Oceanic Technology, 7(1), 157-174.

Weber, B. L., Wuertz, D. D. B., Strauch, R. G., Merritt, D. A., Moran, K. P., Law, D. C., Van de Kamp, D., Chadwick, R. B., Ackley, M., Barth, M., et al. (1990). Preliminary evaluation of the first NOAA demonstration network wind profiler. Journal of atmospheric and oceanic technology, 7(6), 909-918. 